\title{
Early- and late-onset selective fetal growth restriction in monochorionic diamniotic twin pregnancy: natural history and diagnostic criteria
}

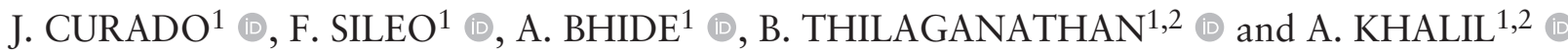 \\ ${ }^{1}$ Fetal Medicine Unit, St George's University Hospitals NHS Foundation Trust, University of London, London, UK; ${ }^{2}$ Vascular Biology \\ Research Centre, Molecular and Clinical Sciences Research Institute, St George's University of London, London, UK
}

KEYWORDS: MCDA twins; morbidity; mortality; natural history; sFGR; superimposed TTTS

\section{CONTRIBUTION}

What are the novel findings of this work?

Using a well-characterized unselected population of monochorionic diamniotic (MCDA) pregnancies, we have provided insight into the natural history of selective fetal growth restriction (sFGR), according to gestational age at onset, as well as the risk of superimposed twin-to-twin transfusion syndrome. Different diagnostic criteria of sFGR were applied and compared in this population.

What are the clinical implications of this work?

This study describes the outcome of a population of MCDA twin pregnancies managed expectantly. The findings provide valuable information for better and more accurate counseling of parents.

\begin{abstract}
Objectives To evaluate the natural history and outcome of selective fetal growth restriction (sFGR) in monochorionic diamniotic (MCDA) twin pregnancy, according to gestational age at onset and various reported diagnostic criteria, and to quantify the risk of superimposed twin-to-twin transfusion syndrome (TTTS).

Methods This was a cohort study of MCDA twin pregnancies that had their routine antenatal care from the first trimester at St George's Hospital, London, UK. Pregnancies had ultrasound examinations every 2 weeks at 16-24 weeks and then every 2-3 weeks until delivery. The diagnostic criteria for sFGR were estimated fetal weight (EFW) of one twin $<10^{\text {th }}$ centile and intertwin EFW discordance $\geq 25 \%$. We also applied other diagnostic criteria reported in a recent Delphi consensus. Pregnancies in which the diagnosis of TTTS was made before that of sFGR were not included in the
\end{abstract}

analysis. Pregnancies that underwent fetal intervention for sFGR were excluded. The incidence of sFGR was compared between the different diagnostic criteria, overall and according to gestational age at onset. In all subsequent analyses, cases of sFGR included those diagnosed according to any of the criteria. The Gratacós classification of sFGR was applied (Type I, II or III). Pregnancy outcomes included miscarriage, intrauterine death, neonatal death and admission to the neonatal unit. Comparisons between groups were carried out using the Mann-Whitney U-test for continuous variables and the chi-square or Fisher's exact test for categorical variables.

Results The analysis included 287 MCDA twin pregnancies. According to the International Society of Ultrasound in Obstetrics and Gynecology diagnostic criteria, the incidence of early (<24 weeks) sFGR was $4.9 \%$, while that of late sFGR was $3.8 \%$. When applying the various diagnostic criteria, the incidence of early sFGR varied from $1.7 \%$ to $9.1 \%$ and that of late sFGR varied from $1.1 \%$ to $5.9 \%$. In early-onset cases, the incidence of Type I sFGR was $80.8 \%$, that of Type II was $15.4 \%$ and that of Type III was $3.8 \%$. The corresponding figures in late-onset cases were $94.4 \%, 5.6 \%$ and $0 \%$. The incidence of superimposed TTTS was $26.9 \%$ in cases affected by early-onset sFGR and $5.6 \%$ in those affected by late-onset sFGR. The incidence of perinatal death was $8.0 \%$ in early-onset sFGR and $5.6 \%$ in late-onset sFGR (P=0.661). Admission to the neonatal unit occurred in $61.0 \%$ and $52.9 \%$ of cases, respectively $(\mathrm{P}=0.484)$.

Conclusions In MCDA twin pregnancies, early-onset sFGR is slightly more common than is late-onset sFGR, although this difference was not significant, and is associated with worse perinatal outcome. The incidence of Types II and III sFGR is higher in early-onset sFGR. The

Correspondence to: Prof. A. Khalil, Fetal Maternal Medicine Unit, St George's University of London, London SW17 0RE, UK (e-mail: akhalil@sgul.ac.uk; asmakhalil79@googlemail.com)

Accepted: 31 July 2019 
incidence also varies according to the diagnostic criteria used, which supports the use of standardized international diagnostic criteria. Superimposed TTTS is more common in early- than in late-onset sFGR. Copyright (c) 2019 ISUOG. Published by John Wiley \& Sons Ltd.

\section{INTRODUCTION}

Twin pregnancy is associated with excess perinatal mortality and morbidity ${ }^{1-6}$. Intensive antenatal fetal surveillance is associated with a lower risk of stillbirth ${ }^{7}$. The excess perinatal mortality and morbidity is higher in monochorionic $(\mathrm{MC})$ than in dichorionic twin pregnancy. This excess mortality is likely to be due to several monochorionicity-related complications, in particular twin-to-twin transfusion syndrome (TTTS) and selective fetal growth restriction $(\mathrm{sFGR})^{8-10}$.

sFGR is relatively common in MC twin pregnancies, with a reported incidence of $12-25 \%^{8-10}$. This condition is thought to be due to unequal placental sharing and to blood flow interchange through placental anastomoses ${ }^{9-15}$. Recently, there has been a focused effort on reaching consensus on the diagnostic criteria, monitoring and management of MC twin pregnancies complicated by $\mathrm{sFGR}^{16}$. However, high-quality studies outlining the natural history of well-characterized cohorts of $\mathrm{MC}$ twin pregnancies complicated by sFGR managed conservatively are scarce.

The old literature suffers from lack of robust discrimination between TTTS, sFGR, TTTS superimposed on sFGR and sFGR following laser treatment for TTTS. There are several other important questions that lack robust answers, such as the incidence of superimposed TTTS in MC twins complicated by sFGR and whether this differs according to gestational age (GA) at onset of $\mathrm{sFGR}^{1}$. Furthermore, studies from tertiary fetal medicine centers might overestimate the incidence of sFGR due to their high-risk populations with referral cases that are often complicated. Moreover, studies that exclude twin pregnancies complicated by intrauterine death (IUD) could also be biased as they could underestimate the incidence of sFGR. Therefore, in order to ascertain the incidence of sFGR, a large cohort of unselected MC twin pregnancies needs to be followed up using regular ultrasound assessment every 2-3 weeks with robust documentation of fetal biometry, amniotic fluid volume and fetal Doppler. The diagnosis of sFGR should be made based on antenatal ultrasound data, which should be confirmed at birth. It is important to acknowledge that the recently published consensus on the diagnostic criteria of sFGR used antenatal ultrasound data, but no postnatal diagnostic criteria were selected to confirm the diagnosis at birth $^{16}$.

The aim of this study was to ascertain the natural history of sFGR in MC twin pregnancies, according to GA at onset and the various reported diagnostic criteria, and to quantify the risk of superimposed TTTS.

\section{METHODS}

This was a longitudinal cohort study of unselected monochorionic diamniotic (MCDA) twin pregnancies that had their routine antenatal care starting in the first trimester at the Fetal Medicine Unit in St George's Hospital, University of London. These pregnancies had regular ultrasound assessment every 2 weeks at 16-24 weeks and then every 2-3 weeks until delivery. Pregnancies were identified retrospectively by searching the ultrasound database (ViewPoint version 5.6.26.148, ViewPoint Bildverarbeitung GMBH, Wessling, Germany). All MCDA twin pregnancies with chorionicity ascertained at 10-14 weeks' gestation between January 2000 and December 2017 were included in this study. Pregnancies complicated by major fetal structural anomaly, aneuploidy or genetic syndrome and those that were lost to follow-up were excluded. Pregnancies in which the diagnosis of TTTS was made before that of sFGR were not included in this analysis. Pregnancies that had an active fetal intervention for sFGR were excluded. We included pregnancies in which all scans were performed in our unit. We obtained pregnancy outcomes from the maternity database and neonatal records.

Twin pregnancies were classified as $\mathrm{MC}$ according to the presence of the T-sign on transabdominal ultrasound examination at 11-14 weeks ${ }^{3}$. GA was determined according to the crown-rump length of the larger fetus ${ }^{17}$ in cases of spontaneous conception, and according to the timing of in-vitro fertilization in pregnancies that underwent assisted reproductive technology. According to the recently published consensus on the diagnostic criteria of sFGR in MC twin pregnancies, one solitary parameter (estimated fetal weight (EFW) of one twin $<3^{\text {rd }}$ centile) was agreed on ${ }^{16}$. Alternatively, at least two out of four contributory parameters (EFW of one twin $<10^{\text {th }}$ centile twin, abdominal circumference (AC) of one twin $<10^{\text {th }}$ centile, EFW discordance $\geq 25 \%$ and umbilical artery (UA) pulsatility index (PI) of smaller twin $>95^{\text {th }}$ centile) were agreed on ${ }^{16}$. We applied these diagnostic criteria in the study population and reported the incidence of sFGR accordingly. A pregnancy was considered to be affected by sFGR if it fulfilled the criteria at two or more scans. According to the published twin pregnancy guideline of the International Society of Ultrasound in Obstetrics and Gynecology (ISUOG), sFGR is diagnosed when EFW of one twin is $<10^{\text {th }}$ centile and $\mathrm{EFW}$ discordance is $\geq 25 \%^{3}$. Intertwin $\mathrm{EFW}$ discordance was calculated as the difference in EFW divided by the EFW of the large twin. EFW prior to 20 weeks was derived using the formula of Warsof et al. ${ }^{18}$, and at 20 weeks or beyond using the formula of Hadlock et al. ${ }^{19}$. Growth was evaluated using the STORK twin growth charts $^{20}$. In order to compare the performance of these diagnostic criteria, we defined seven different groups using different combinations of criteria adapted from Khalil et al. ${ }^{16}$ (Table 1). In all subsequent analyses, cases of sFGR included those diagnosed according to any of the criteria.

Doppler assessment of the UAs of both twins and classification of end-diastolic flow (EDF) as normal, absent or reversed was carried out in all cases at every 
Table 1 Incidence of selective fetal growth restriction (sFGR) in 287 monochorionic diamniotic twin pregnancies, according to different diagnostic criteria in consensus definition of Khalil et al. ${ }^{16}$ and gestational age (GA) at onset

\begin{tabular}{|c|c|c|c|c|c|}
\hline \multirow[b]{2}{*}{ Diagnostic criterion } & \multirow[b]{2}{*}{$\begin{array}{l}\text { Incidence of } \\
\text { all sFGR (\%) }\end{array}$} & \multicolumn{2}{|c|}{ Early-onset sFGR } & \multicolumn{2}{|c|}{ Late-onset sFGR } \\
\hline & & $\begin{array}{l}\text { Incidence } \\
(\%)\end{array}$ & $\begin{array}{l}\text { GA at diagnosis } \\
\text { (weeks) }\end{array}$ & $\begin{array}{l}\text { Incidence } \\
(\%)\end{array}$ & $\begin{array}{c}\text { GA at diagnosis } \\
\text { (weeks) }\end{array}$ \\
\hline EFW of one twin $<3^{\text {rd }}$ centile & 8.7 & 5.6 & $18.5(17-21)$ & 3.1 & $31(30-33)$ \\
\hline $\begin{array}{l}\text { EFW of one twin }<10^{\text {th }} \text { centile } \\
\quad+\text { EFW discordance } \geq 25 \%\end{array}$ & 8.7 & 4.9 & $20(18-24)$ & 3.8 & $31(29-32)$ \\
\hline $\begin{array}{l}\text { EFW of one twin }<10^{\text {th }} \text { centile } \\
\quad+\text { UA-PI of smaller twin }>95^{\text {th }} \text { centile }\end{array}$ & 2.8 & 1.7 & $20.5(17-24)$ & 1.1 & $32(28-32)$ \\
\hline AC of one twin $<10^{\text {th }}$ centile & 15.0 & 9.1 & $18(16-20)$ & 5.9 & $30(27-33)$ \\
\hline $\begin{array}{l}\text { AC of one twin }<10^{\text {th }} \text { centile } \\
\quad+\text { EFW discordance } \geq 25 \%\end{array}$ & 8.0 & 4.9 & $18(17-23)$ & 3.1 & $31(29-33)$ \\
\hline $\begin{array}{l}\text { AC of one twin }<10^{\text {th }} \text { centile } \\
\quad+\text { UA-PI of smaller twin }>95^{\text {th }} \text { centile }\end{array}$ & 4.9 & 3.8 & $19(16-29)$ & 1.1 & $30(28-32)$ \\
\hline $\begin{array}{l}\text { EFW discordance } \geq 25 \% \\
\quad+\text { UA-PI of smaller twin }>95^{\text {th }} \text { centile }\end{array}$ & 3.8 & 1.7 & $21(18-30)$ & 2.1 & $32(28-32)$ \\
\hline
\end{tabular}

GA data are given as median (range). AC, abdominal circumference; EFW, estimated fetal weight; PI, pulsatility index; UA, umbilical artery.

scan. According to Gratacós et al., sFGR is subdivided into Types I, II and III according to the Doppler finding of $\mathrm{EDF}$ in the UA of the smaller fetus, which is normal in Type I, absent or reversed (AREDF) in Type II or intermittent AREDF in Type III ${ }^{11}$. We categorized our sFGR population as Type I, II or III according to the type at diagnosis. Since GA at viability in the UK is 24 weeks and as there are various cut-offs used in the literature (20-26 weeks), we defined early-onset sFGR as diagnosis $\leq 24$ weeks' gestation and late-onset sFGR as diagnosis $>24$ weeks' gestation ${ }^{8}$. Miscarriage was defined as death of at least one twin $<20$ weeks' gestation and IUD as death from 20 weeks onwards. Neonatal death was defined as death of at least one neonate up to 28 days postpartum. Perinatal death was defined as the sum of IUD and neonatal death.

\section{Statistical analysis}

Continuous variables are presented as mean and standard deviation or median and interquartile range, while the categorical variables are presented as $n(\%)$. Comparisons between groups were performed using the Mann-Whitney $U$-test for continuous variables or the chi-square or Fisher's exact test for categorical variables. Differences between groups were considered significant at a $P$-value of $<0.05$ using two-tailed tests. Analysis was performed using SPSS version 22 (IBM Corp., Armonk, NY, USA).

\section{RESULTS}

\section{Study population}

The study population included 315 MCDA twin pregnancies. Eleven pregnancies were excluded due to fetal anomalies (including four cases of twin reversed arterial perfusion sequence, two cases of acrania, one of spina bifida, one of tetralogy of Fallot, one of left isomerism, one of hypoplastic left heart syndrome and one of pulmonary stenosis). Of the remaining pregnancies, the diagnosis of TTTS was made in 15 not yet affected by sFGR (10 later fulfilled the criteria for sFGR), and these were therefore not included in the analysis. Two pregnancies affected by sFGR were further excluded from the analysis because the parents opted for an invasive procedure (one had laser and cord occlusion at 23 weeks and the other had laser at 20 weeks) in an attempt to improve the prognosis. The remaining 287 MCDA twin pregnancies were included in the analysis (Figure 1). Perinatal outcome was available in $98 \%$ of pregnancies affected by sFGR and in $94 \%$ of those with concordant growth. Table 2 shows the baseline characteristics of the study population, which were similar between pregnancies with sFGR and those with concordant growth.

\section{Incidence of sFGR}

According to the ISUOG diagnostic criteria (EFW of one twin $<10^{\text {th }}$ centile and EFW discordance $\geq 25 \%$ ), the incidence of sFGR overall was $8.7 \%(n=25)$, while that of early-onset sFGR was $4.9 \%(n=14)$ and that of late-onset sFGR was $3.8 \%(n=11)$. Of those pregnancies diagnosed with sFGR, 56\% were early onset and $44 \%$ were late onset $(P=0.63)$. Table 1 shows the incidence of sFGR and median GA at which sFGR was first diagnosed, according to each proposed diagnostic criterion. The incidence varied between $2.8 \%$ when using EFW $<10^{\text {th }}$ centile with UA-PI $>95^{\text {th }}$ centile, and $15.0 \%$ using AC $<10^{\text {th }}$ centile. The incidence was similar when using EFW $<3^{\text {rd }}$ centile, EFW $<10^{\text {th }}$ centile with intertwin EFW discordance $\geq 25 \%$ or $\mathrm{AC}<10^{\text {th }}$ centile with intertwin EFW discordance $\geq 25 \%$. Regardless of the diagnostic criteria used, most cases of sFGR were early onset. In total, 44 pregnancies were found to be affected by sFGR when considering the various diagnostic criteria (Table 1). 


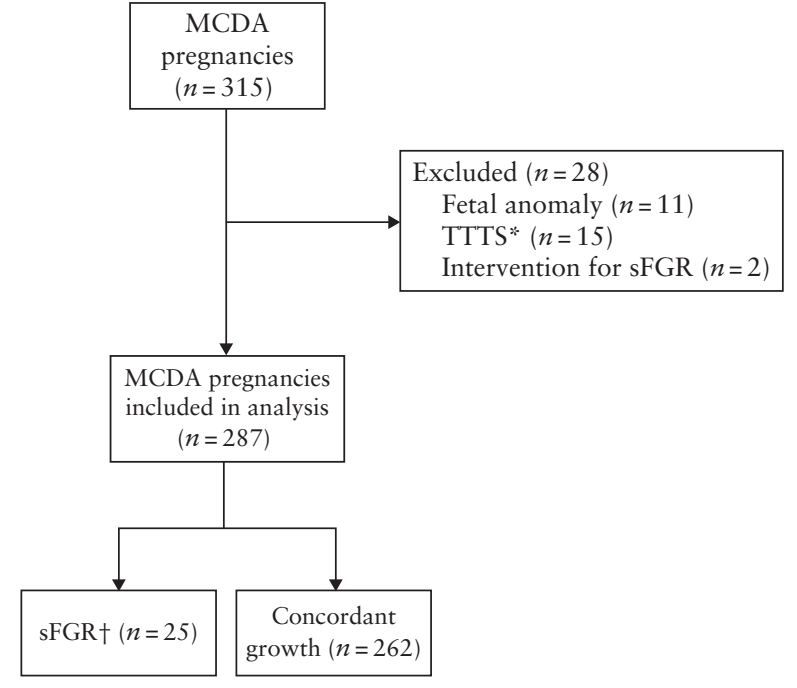

Figure 1 Flowchart summarizing inclusion of study population of monochorionic diamniotic (MCDA) twin pregnancies. "Excluded from sFGR group only if diagnosis made before that of sFGR. †According to International Society of Ultrasound in Obstetrics and Gynecology diagnostic criteria for $\mathrm{sFGR}^{3}$. sFGR, selective fetal growth restriction; TTTS, twin-to-twin transfusion syndrome.

Table 2 Baseline characteristics of 287 monochorionic diamniotic twin pregnancies, according to whether pregnancy was complicated by selective fetal growth restriction (sFGR)

\begin{tabular}{lccc}
\hline Characteristic & $\begin{array}{c}s F G R \\
(\mathrm{n}=44)\end{array}$ & $\begin{array}{c}\text { No sFGR } \\
(\mathrm{n}=243)\end{array}$ & $\mathrm{P}$ \\
\hline Maternal age (years) & $32(27-36)$ & $32(28-35)$ & 0.640 \\
BMI $\left(\mathrm{kg} / \mathrm{m}^{2}\right)$ & $24.7(21.0-26.8)$ & $23.9(21.7-26.9)$ & 0.851 \\
Ethnicity & $31(70.5)$ & $163(67.1)$ & 0.625 \\
$\quad$ Caucasian & $3(6.8)$ & $25(10.3)$ & \\
Afro-Caribbean & $8(18.2)$ & $34(14.0)$ & \\
South Asian & $0(0)$ & $8(3.3)$ & \\
East Asian & $0(0)$ & $5(2.1)$ & \\
Mixed & $2(4.5)$ & $8(3.3)$ & \\
$\quad$ Other & $29(65.9)$ & $132(54.3)$ & 0.171 \\
Nulliparous &
\end{tabular}

Data are given as median (interquartile range) or $n(\%)$. Diagnosis of sFGR was according to any criterion in Table 1 . BMI, body mass index.

\section{Gratacós classification of sFGR}

Type I sFGR was the most common in both early-onset $(80.8 \%, n=21)$ and late-onset $(94.4 \%, n=17)$ cases. In early-onset cases, the incidence of Type II sFGR was $15.4 \%$ and that of Type III sFGR was $3.8 \%$. All cases of late-onset sFGR were Type I except one $(5.6 \%)$, which was Type II according Gratacós et al. None of the cases in the late-onset sFGR group was Type III sFGR.

\section{Superimposed TTTS}

The incidence of superimposed TTTS was $26.9 \%(n=7)$ in cases affected by early-onset sFGR and $5.6 \%(n=1)$ in those affected by late-onset sFGR $(P=0.07)$. The incidence of superimposed TTTS was $13.2 \%(n=5)$ in pregnancies affected by Type I sFGR and $60.0 \%(n=3)$ in those affected by Type II sFGR $(P=0.034)$. None in the Type III sFGR group had superimposed TTTS. Four cases of superimposed TTTS (all in the early-onset sFGR group) underwent fetoscopic laser ablation.

\section{Pregnancy outcome}

Delivery occurred significantly earlier in MCDA twin pregnancies complicated by sFGR compared with those with concordant growth (34 vs 36 weeks, $P<0.001$; Table 3$)$. IUD was significantly more common in twin pregnancies with sFGR $(7.0 \%$ vs $1.8 \%, P=0.005)$. The incidence of PND was more than doubled in pregnancies complicated by sFGR, although this difference was not significant $(7.0 \%$ vs $2.9 \%, P=0.056)$. Admission to the neonatal unit (NNU) was also more frequent in those with sFGR than in those with concordant growth $(57.3 \%$ vs $23.0 \%, P<0.001$ ).

Delivery occurred significantly earlier in early-onset compared with late-onset sFGR pregnancies (33 vs 34 weeks, $P=0.006$; Table 3 ). Early fetal loss occurred in $4 \%$ of pregnancies with early-onset sFGR and in none of those with late-onset sFGR. There were no cases of neonatal death in the sFGR group. Although there were

Table 3 Pregnancy outcome and perinatal mortality in 574 monochorionic diamniotic twins, according to whether pregnancy was complicated by selective fetal growth restriction (sFGR), and gestational age at onset

\begin{tabular}{|c|c|c|c|c|c|c|}
\hline \multirow[b]{2}{*}{ Outcome } & \multirow[b]{2}{*}{$\begin{array}{c}s F G R \\
(\mathrm{n}=88)\end{array}$} & \multirow[b]{2}{*}{$\begin{array}{l}\text { No sFGR } \\
(\mathrm{n}=486)\end{array}$} & \multirow[b]{2}{*}{$\mathrm{P}$} & \multicolumn{3}{|c|}{ Onset of sFGR } \\
\hline & & & & $\begin{array}{c}\text { Early } \\
(\mathrm{n}=52)\end{array}$ & $\begin{array}{c}\text { Late } \\
(\mathrm{n}=36)\end{array}$ & $\mathrm{P}$ \\
\hline Gestational age at birth (weeks) & $34(31-36) *$ & $36(34-37) \dagger$ & $<0.001$ & $33(30-36) \ddagger$ & $34(33-37) \mathbb{S}$ & 0.006 \\
\hline Birth weight $(\mathrm{g})$ & $1832 \pm 568^{*}$ & $2293 \pm 529 \dagger$ & $<0.001$ & $1694 \pm 524 \ddagger$ & $2006 \pm 581 \rrbracket$ & 0.049 \\
\hline Birth-weight centile & $30.7 \pm 29.0 *$ & $50.7 \pm 25.0+$ & $<0.001$ & $27.8 \pm 24.3 \ddagger$ & $34.4 \pm 34.0 \mathbb{S}$ & 0.682 \\
\hline Miscarriage & $2 / 86(2.3)$ & $27 / 456(5.9)$ & 0.064 & $2 / 50(4.0)$ & $0 / 36(0.0)$ & 0.475 \\
\hline Intrauterine death & $6 / 86(7.0)$ & $8 / 456(1.8)$ & 0.005 & $4 / 50(8.0)$ & $2 / 36(5.6)$ & 0.661 \\
\hline Neonatal death & $0 / 86(0.0)$ & $5 / 456(1.1)$ & 0.329 & $0 / 50(0.0)$ & $0 / 36(0.0)$ & - \\
\hline Perinatal death & $6 / 86(7.0)$ & $13 / 456(2.9)$ & 0.056 & $4 / 50(8.0)$ & $2 / 36(5.6)$ & 0.661 \\
\hline Survival of at least one twind & $40 / 43(93.0)$ & $219 / 228(95.1)$ & 0.179 & $22 / 25(88.0)$ & $17 / 18(94.4)$ & 0.756 \\
\hline Survival of both twins $\mathbb{1}$ & $39 / 43(90.7)$ & $218 / 228(95.6)$ & 0.410 & $20 / 25(80.0)$ & $17 / 18(94.4)$ & 0.177 \\
\hline Neonatal unit admission & $43 / 75(57.3)$ & $90 / 392(23.0)$ & $<0.001$ & $25 / 41(61.0)$ & $18 / 34(52.9)$ & 0.484 \\
\hline
\end{tabular}

Data are given as median (interquartile range), mean \pm SD or $n / N(\%)$. Diagnosis of sFGR was according to any criteria in Table 1 . Perinatal outcome was not available for all fetuses/neonates due to loss to follow-up. Data available in: *86 neonates; †456 neonates; $\ddagger 50$ neonates; $\$ 36$ neontates. đIDenominators are number of pregnancies. 
more NNU admissions $(61.0 \%$ vs $52.9 \%, P=0.484)$ and more IUDs $(8.0 \%$ vs $5.6 \%, P=0.661)$ in the early-onset than in the late-onset sFGR group, the difference was not statistically significant.

\section{DISCUSSION}

\section{Summary of study findings}

According to the ISUOG diagnostic criteria $\left(\mathrm{EFW}<10^{\text {th }}\right.$ centile in one twin and EFW discordance $\geq 25 \%$ ), the incidence of sFGR in this population of MCDA twin pregnancies was $8.7 \%$. Early-onset sFGR was slightly more common than late-onset $\mathrm{sFGR}(4.9 \%$ vs $3.8 \%)$; however, this difference was not statistically significant. Types II and III sFGR and superimposed TTTS were more common in early-onset sFGR. MCDA twin pregnancies complicated by sFGR were associated with a higher rate of perinatal mortality and admission to the NNU compared with those with concordant growth.

\section{Interpretation of study findings and comparison with existing literature}

Early-onset sFGR is expected to have the highest rate of perinatal mortality ${ }^{21}$ ( $8.0 \%$ in our study). These twins typically have an unequally shared placenta with large anastomoses ${ }^{22}$. According to Lewi et al. ${ }^{22}$, IUD occurs in about $20 \%$ of cases of early-onset sFGR and most have abnormal UA Doppler from 16 weeks. In our study, IUD occurred in $8.0 \%$ of fetuses in the early-onset group, and only $19.2 \%$ had abnormal UA Doppler at diagnosis. According to Lewi et al. ${ }^{22}$, the UA of the smaller twin is always normal in late-onset sFGR and the survival rate is nearly $100 \%$. In our study, the survival rate was $94.5 \%$ (due to two cases with IUD after 24 weeks' gestation) and one case was diagnosed with Type II sFGR at 32 weeks. One possible explanation for these differences is the fact that Lewi et al. included data on discordant growth, rather than sFGR. Moreover, they used a GA cut-off of 20 weeks to define early onset and 26 weeks' gestation for late onset. Consequently, the early-onset group likely included more severe cases than did ours and the late-onset group likely included milder cases.

Gratacós et al. classified sFGR into three types, each of which has a different clinical evolution and perinatal outcome $^{11,12,23}$. In our study, Type I sFGR was the most frequent in both early- and late-onset sFGR, accounting for $80.8 \%$ and $94.4 \%$ of cases, respectively. Type II was more frequent in early-onset $(15.4 \%)$ compared with late-onset $(5.6 \%)$ sFGR. Type III was seen in only one case of early-onset sFGR, whilst it was not seen in any case of late-onset sFGR.

The inclusion of intertwin size discordance in the diagnostic criteria for sFGR reduces the variation introduced by the use of fetal biometry, with the limitation of which equation was used and whether a singleton or twin-specific chart was applied. These are known sources of variation in ultrasound assessment of fetal size. The incidence of sFGR $(8.7 \%$ vs $8.0 \%)$, both early onset $(4.9 \%$ vs $4.9 \%)$ and late onset $(3.8 \%$ vs $3.1 \%)$, was similar regardless of whether EFW or $\mathrm{AC}<10^{\text {th }}$ centile was used, as long as intertwin EFW discordance $\geq 25 \%$ was included in the diagnostic criteria. The use of EFW $<3^{\text {rd }}$ centile alone yielded a similar incidence compared with $\mathrm{AC}<10^{\text {th }}$ centile in association with EFW discordance $\geq 25 \%$, and $\mathrm{EFW}<10^{\text {th }}$ centile with EFW discordance $\geq 25 \%$. Interestingly, this supports the recently published consensus diagnostic criteria of sFGR in $\mathrm{MC}$ twin pregnancies ${ }^{16}$. The use of these criteria in association with UA-PI $>95^{\text {th }}$ centile is likely to identify a more severe subset of sFGR.

In our population, sFGR was slightly more common before 24 weeks' gestation, but the difference was not statistically significant. This is different from dichorionic twins, in which early-onset FGR is less common than late-onset $\mathrm{sFGR}^{24}$. The higher incidence of superimposed TTTS in early-onset than in late-onset sFGR could reflect the higher risk of TTTS prior to 24 weeks and reiterates the observation that late-onset TTTS is uncommon and is usually acute in nature.

\section{Clinical and research implications}

Prenatal counseling of parents with MC twin pregnancy affected by sFGR is challenging due to the lack of robust data from large prospective studies. Most of the published studies are retrospective, with small numbers and heterogeneous diagnostic criteria and management. Because the diagnostic criteria used in these studies vary, it is difficult to compare pregnancy outcomes or combine data in a meta-analysis.

The optimal management of MC twins with sFGR is not yet defined. Expectant management, elective preterm birth, fetoscopic laser coagulation of the vascular anastomoses and selective fetocide are valid options $^{10,25}$. Furthermore, reporting of fetoscopy- and laser-surgery-related complications is lacking ${ }^{26}$. According to a recent systematic review, expectant management is appropriate in most cases of Type I sFGR. For Types II and III sFGR diagnosed before 24 weeks, fetal therapy may contribute to higher mortality with lower morbidity of the surviving twin ${ }^{2}$. Although perinatal mortality in multiple pregnancy seems to be decreasing, the rate of stillbirth is still much higher compared with that in singletons ${ }^{27}$.

\section{Strengths and limitations}

Our study reports the incidence of sFGR, its types, superimposed TTTS and perinatal mortality in these pregnancies according to $\mathrm{GA}$ at diagnosis. It provides valuable information for more accurate counseling of parents. The main limitation of this study is its retrospective design and the number of pregnancies included. Moreover, we did not use the total combination of contributory parameters proposed for the diagnosis of sFGR in MCDA pregnancies according to the Delphi procedure 
by Khalil et al. According to this consensus, different solitary and contributory parameters were proposed in 12 different combinations (EFW $<3^{\text {rd }}$ centile alone; at least two of: EFW $<10^{\text {th }}$ centile, EFW discordance $\geq 25 \%$, UA-PI $>95^{\text {th }}$ percentile and AC $<10^{\text {th }}$ centile $)^{16}$.

The management options have not changed over the study period due to the lack of robust evidence or guidance. Two cases had active fetal intervention for sFGR (laser and cord occlusion) and were excluded from the analysis. Eight pregnancies had superimposed TTTS, of which half underwent fetoscopic laser ablation of anastomoses, which could have modified the natural history of sFGR in these pregnancies.

\section{Conclusions}

In MCDA twin pregnancies, early-onset sFGR is slightly more common than late-onset sFGR and seems to be associated with worse perinatal outcome. Superimposed TTTS and Types II and III sFGR are more common in early-onset than in late-onset sFGR. Future large multicenter studies with well-characterized MCDA cohorts are needed in order to assess the performance of the various diagnostic criteria in defining the severity of sFGR, as well as establishing perinatal outcome in these pregnancies.

\section{REFERENCES}

1. Khalil A, Thilaganathan B. Selective fetal growth restriction in a monochorionic twin pregnancy: a dilemma for clinicians and a challenge for researchers. Ultrasound Obstet Gynecol 2019; 53: 23-25.

2. Townsend R, D'Antonio F, Sileo FG, Kumbay H, Thilaganathan B, Khalil A. Perinatal outcome of monochorionic twin pregnancies complicated by selective fetal growth restriction according to management: systematic review and meta-analysis. Ultrasound Obstet Gynecol 2019; 53: 36-46.

3. Khalil A, Rodgers M, Baschat A, Bhide A, Gratacós E, Hecher K, Kilby MD, Lewi L, Nicolaides KH, Oepkes D, Raine-Fenning N, Reed K, Salomon LJ, Sotiriadis A, Thilaganathan B, Ville Y. ISUOG Practice Guidelines: role of ultrasound in twin pregnancy. Ultrasound Obstet Gynecol 2016; 47: 247-263.

4. Danon D, Sekar R, Hack KE, Fisk NM. Increased stillbirth in uncomplicated monochorionic twin pregnancies: a systematic review and meta-analysis. Obstet Gynecol 2013; 121: 1318-1326.

5. Cheong-See F, Schuit E, Arroyo-Manzano D, Khalil A, Barrett J, Joseph KS, Asztalos E, Hack K, Lewi L, Lim A, Liem S, Norman J, Morrison J, Combs CA, Garite TJ, Maurel K, Serra V, Perales A, Rode L, Worda K, Nassar A, Aboulghar M, Rouse D, Thom E, Breathnach F, Nakayama S, Russo FM, Robinson JN, Dodd JM, Newman RB, Bhattacharya S, Tang S, Mol B W J, Zamora J, Thilaganathan B, Thangaratinam S; Global Obstetrics Network (GONet) Collaboration. Prospective risk of stillbirth and neonatal complications in twin pregnancies: systematic review and meta-analysis. BMJ 2016; 354: 14353.
6. Lee YM, Wylie BJ, Simpson LL, D'Alton ME. Twin chorionicity and the risk of stillbirth. Obstet Gynecol 2008; 111: 301-308.

7. Burgess JL, Unal ER, Nietert PJ, Newman RB. Risk of late-preterm stillbirth and neonatal morbidity for monochorionic and dichorionic twins. Am J Obstet Gynecol 2014; 210: 578.e1-9.

8. Lewi L, Gucciardo L, van Mieghem T, de Koninck P, Beck V, Medek H, Van Schoubroeck D, Devlieger R, De Catte L, Deprest J. Monochorionic diamniotic twin pregnancies: natural history and risk stratification. Fetal Diagn Ther 2010; 27: 121-133.

9. Valsky DV, Eixarch E, Martinez JM, Crispi F, Gratacós E. Selective intrauterine growth restriction in monochorionic twins: pathophysiology, diagnostic approach and management dilemmas. Semin Fetal Neonatal Med 2010; 15: 342-348.

10. Inklaar MJ, van Klink JM, Stolk TT, van Zwet EW, Oepkes D, Lopriore E. Cerebral injury in monochorionic twins with selective intrauterine growth restriction: a systematic review. Prenat Diagn 2014; 34: 205-213.

11. Gratacós E, Lewi L, Munoz M, Acosta-Rojas R, Hernandez-Andrade E, Martinez JM, Carreras E, Deprest J. A classification system for selective intrauterine growth restriction in monochorionic pregnancies according to umbilical artery Doppler flow in the smaller twin. Ultrasound Obstet Gynecol 2007; 30: 28-34.

12. Townsend R, Khalil A. Twin pregnancy complicated by selective growth restriction. Curr Opin Obstet Gynecol 2016; 28: 485-491.

13. Buca D, Pagani G, Rizzo G, Familiari A, Flacco ME, Manzoli L, Liberati M, Fanfani F, Scambia G, D'Antonio F. Outcome of monochorionic twin pregnancy with selective intrauterine growth restriction according to umbilical artery Doppler flow pattern of smaller twin: systematic review and meta-analysis. Ultrasound Obstet Gynecol 2017; 50: 559-568.

14. Valsky DV, Eixarch E, Martinez JM; Gratacós E. Selective intrauterine growth restriction in monochorionic diamniotic twin pregnancies. Prenat Diagn 2010; 30: $719-726$.

15. Lewi L, Gucciardo L, Huber A, Jani J, Van Mieghem T, Doné E, Cannie M, Gratacós E, Diemert A, Hecher K, Lewi P, Deprest J. Clinical outcome and placental characteristics of monochorionic diamniotic twin pairs with early- and late-onset discordant growth. Am J Obstet Gynecol 2008; 199: 511.e1-7.

16. Khalil A, Beune I, Hecher K, Wynia K, Ganzevoort W, Reed K, Lewi L, Oepkes D, Gratacós E, Thilaganathan B, Gordijn SJ. Consensus definition and essential reporting parameters of selective fetal growth restriction in twin pregnancy: a Delphi procedure. Ultrasound Obstet Gynecol 2019; 53: 47-54.

17. Robinson HP, Fleming JE. A critical evaluation of sonar crown rump length measurements. Br J Obstet Gynaecol 1975; 82: 702-710.

18. Warsof SL, Gohari P, Berkowitz RL, Hobbins JC. The estimation of fetal weight by computer-assisted analysis. Am J Obstet Gynecol 1977; 128: 881-892.

19. Hadlock FP, Harrist RB, Martinez-Poyer J. In utero analysis of fetal growth: a sonographic weight standard. Radiology 1991; 181: 129-133.

20. Stirrup OT, Khalil A, D'Antonio F, Thilaganathan B. Fetal growth reference ranges in twin pregnancy: analysis of the Southwest Thames Obstetric Research Collaborative (STORK) multiple pregnancy cohort. Ultrasound Obstet Gynecol 2015; 45: 301-307.

21. Bennassar M, Eixarch E, Martinez JM, Gratacós E. Selective intrauterine growth restriction in monochorionic diamniotic twin pregnancies. Semin Fetal Neonatal Med 2017; 22: 376-382.

22. Lewi L, Deprest J, Hecher K. Vascular anastomoses in monochorionic twin pregnancies and their clinical consequences. Am J Obstet Gynecol 2013; 208 $19-30$.

23. Monaghan C, Kalafat E, Binder J, Thilaganathan B, Khalil A. Prediction of adverse pregnancy outcome in monochorionic-diamniotic twin pregnancies complicated by selective fetal growth restriction. Ultrasound Obstet Gynecol 2019; 53: 200-207.

24. Sebire NJ, D'Ercole C, Hughes K, Rennie J, Nicolaides KH. Dichorionic twins discordant for intrauterine growth retardation. Arch Dis Child Fetal Neonatal Ed 1997; 77: F235-F236.

25. Quintero RA, Bornick PW, Morales WJ, Allen MH. Selective photocoagulation of communicating vessels in the treatment of monochorionic twins with selective growth retardation. Am J Obstet Gynecol 2001, 185: 689-696.

26. Sileo FG, Duffy JMN, Townsend R, Khalil A. Variation in outcome reporting acros studies evaluating interventions for selective fetal growth restriction. Ultrasound Obstet Gynecol 2019; 54: 10-15.

27. Khalil A. Unprecedented fall in stillbirth and neonatal deaths in twins: lessons from the United Kingdom. Ultrasound Obstet Gynecol 2019; 53: 153-157. 